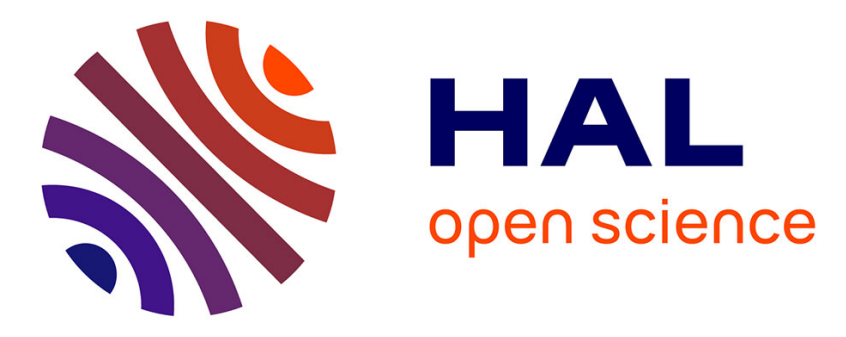

\title{
Neural correlates of interoceptive accuracy: Beyond cardioception
}

Matias Baltazar, Julie Grezes, Marie-maude Geoffray, Jean-luc Picq, Laurence Conty

\section{- To cite this version:}

Matias Baltazar, Julie Grezes, Marie-maude Geoffray, Jean-luc Picq, Laurence Conty. Neural correlates of interoceptive accuracy: Beyond cardioception. European Journal of Neuroscience, 2021, 10.1111/ejn.15510 . hal-03432796

\section{HAL Id: hal-03432796 https://hal.science/hal-03432796}

Submitted on 17 Nov 2021

HAL is a multi-disciplinary open access archive for the deposit and dissemination of scientific research documents, whether they are published or not. The documents may come from teaching and research institutions in France or abroad, or from public or private research centers.
L'archive ouverte pluridisciplinaire HAL, est destinée au dépôt et à la diffusion de documents scientifiques de niveau recherche, publiés ou non, émanant des établissements d'enseignement et de recherche français ou étrangers, des laboratoires publics ou privés. 


\title{
NEURAL CORRELATES OF INTEROCEPTIVE ACCURACY: BEYOND CARDIOCEPTION
}

\author{
Matias BALTAZAR ${ }^{1}$, Julie GREZES ${ }^{2}$, Marie-Maude GEOFFRAY ${ }^{1,3}$, Jean-Luc PICQ ${ }^{4}$ and \\ Laurence CONTY ${ }^{4}$ \\ ${ }^{\text {I}}$ Centre d'Evaluation et de Diagnostic de l'Autisme (CEDA) - Centre Hospitalier Le Vinatier, Bron Cedex, \\ France
}

${ }^{2}$ Cognitive and Computational Neuroscience Laboratory, INSERM unit 960, Ecole Normale Supérieure, PSL University, Paris, 75005, France

${ }^{3}$ Health Services and Performance Research EA7425, Claude Bernard Lyon 1 University (CBL1), Lyon, France

${ }^{4}$ Laboratory of Cognitive Functioning and Dysfunctioning (DysCo), Univ. Paris Nanterre, F92000 Nanterre, France.

Journal: European Journal of Neuroscience

Journal section: Research Paper

Published: 29 October 2021

DOI: https://doi.org/10.1111/ejn.15510

*Corresponding author:

Matias Baltazar

Centre d'Evaluation et de Diagnostic de l'Autisme

CH le Vinatier Bât 504B

95 boulevard Pinel

69678 BRON

FRANCE 
Abstract (250 words max): Interoceptive Accuracy (IAc), the precision with which one assesses the signals arising from one's own body, is receiving increasing attention in the literature. IAc has mainly been approached as an individual trait and has been investigated through the cardiac modality using mostly non ecological methods. Such studies consensually designate the anterior insular cortex as the main brain correlate of IAc. However, there is a lack of brain imaging studies investigating IAc in a broader and more ecological way. Here, we used a novel ecological task in which participants monitored their general bodily reactions to external events and investigated brain regions subtending intra-individual (i.e. trial-by-trial) variations of IAc. At each trial, participants had to rate the intensity of their bodily reactions to an emotional picture. We recorded participants' skin conductance response (SCR) to the picture as an indicator of actual physiological response intensity. We fitted an fMRI model using, as regressors, the SCR value, the rating, and the product of the two (as a proxy of participants' IAc) obtained trial per trial. We observed that activity in the dorsomedial prefrontal cortex (dmPFC) increased when individuals' IAc decreased. This result reveals general mechanisms of error processing in intra-individual variations of IAc, which are unspecific to interoception. Our result has a practical impact in the clinical domain. Namely, it supports the predictive coding framework whereby IAc deficits may reflect impairments in processing a mismatch between actual interoceptive signals and predictions.

Keywords: interoception, fMRI, skin conductance, emotion, medial prefrontal cortex.

\section{Highlights:}

- Interoceptive accuracy is the ability to assess one's own bodily signals accurately

- The question of the neural basis of interoceptive accuracy is under investigated

- We investigate this question with an ecological task using fMRI

- We show a key role of the dorsomedial prefrontal cortex

- Interoceptive accuracy may rely on unspecific error processing mechanisms 


\section{Introduction}

Interoception is the sensing of the physiological state of the body (Craig, 2009). It is crucial to maintain internal homeostasis and helps make decisions regarding external stimuli based on actual or anticipated bodily reactions to those stimuli (Carvalho \& Damasio, 2021; Chen et al., 2021; Craig, 2009). Interoceptive accuracy (IAc) is one dimension of interoception and designates the ability to accurately detect and track internal bodily sensations, which can be objectively quantified using behavioral performance (Garfinkel et al., 2015). Quantifying IAc implies measuring interoceptive signals (e.g., heartbeats), recording individual reports regarding these signals (e.g., deciding whether external sounds are synchronized with our own heartbeats), and evaluate the accuracy of the reports by confronting them with the interoceptive signals. IAc is suggested to be the central construct of interoception, underpinning the other two dimensions, namely, interoceptive sensibility (i.e. the individual's belief that he/she is interoceptively focused, assessed via self-questionnaires) and interoceptive awareness (i.e. the metacognitive ability to assess one's own IAc (Garfinkel et al., 2015)).

Traditional research in human cognition tends to isolate cognitive processes from natural contexts. Thus, most studies on the neural correlates of IAc instruct participants to focus on a specific interoceptive signal (e.g., heartbeat or breathing) at rest (i.e., in the absence of external events that would induce bodily reactions). Such a method contradicts the view that cognition is necessarily embedded in a context (e.g. (Ward \& Stapleton, 2012)). In this respect, monitoring bodily states is involved in the attribution of value to external objects that induce bodily changes, to further make appropriate decisions towards these objects (Carvalho \& Damasio, 2021; Hazem et al., 2018). Thus, traditional tasks used to investigate the neural bases of IAc can be considered somewhat artificial. It is also noteworthy that different interoceptive modalities (e.g., muscle tension, temperature, breath) do not share common 
sensory pathways and are therefore likely to be treated differently in the brain. For instance, recent data show a dissociation in interoceptive accuracy between cardiac and respiratory modalities (Garfinkel et al., 2016), which may imply different types of IAc processing in the brain. Finally, the validity of the heartbeat counting task, which is the most commonly used method to investigate the neural basis of IAc, is seriously questioned, with growing evidence showing that this task mainly addresses beliefs about heart rates rather than actual heart beat monitoring (Desmedt et al., 2018; Murphy et al., 2018; Ring et al., 2015; Zamariola et al., 2018). Altogether, these different arguments call for testing the reproducibility of previous results using new and more ecological tasks to assess the neural correlates of IAc.

Several pieces of evidence converge toward the view that the right insula is involved in IAc. One seminal study investigating the neural bases of IAc showed that activity in the right anterior insula correlated with performance accuracy on a heartbeat detection task (i.e. detecting whether there is a delay between one's heartbeats and synchronous or asynchronous tones (Critchley et al., 2004)). Another study that explored the covariation between IAc (measured as the discrepancy between the actual and perceived number of heartbeats counted by participants during time intervals) and brain activity during cardiac interoceptive attention identified the right insula, medial frontal gyrus and cingulate gyrus as critical areas (Pollatos et al., 2007). Another study found a significant correlation between activity in the right insula and better performance at a heartbeat tracking task, using an ROI approach (Caseras et al., 2013). Note that in all these studies, correlations between IAc and brain activity were calculated at the group level, revealing brain markers of good heartbeat perceivers, which fits the conceptualization of IAc as a stable individual trait (Ferentzi et al., 2018; Wittkamp et al., 2018). However, as mentioned above, in daily life, processes underlying IAc occur in a large variety of contexts that can impact individual IAc ability. Stressful contexts for example, or self-reflexive contexts (i.e. being reminded of oneself) may affect individuals' IAc (e.g.(Ainley et al., 2013; Durlik et al., 2014)). Here, using a more ecological task in which 
participants monitored their internal states in response to external events, we aimed at identifying the brain mechanisms subtending intra-individual variations of IAc in a trial-pertrial fashion.

To further our knowledge of IAc beyond cardioception or another specific interoceptive modality, we used a paradigm developed by our team in which participants are presented with an emotional image at each trial and are asked to evaluate the intensity of their emotional reaction to the image based on their physiological changes (Baltazar et al., 2014). To induce variation in participants' emotional experience, we manipulated the arousing power of the images. We also used context pictures pertaining to different levels of social contact at the beginning of each trial to manipulate interoceptive accuracy, since we previously showed that social contact increases interoceptive accuracy (Baltazar et al., 2014; Hazem et al., 2017, 2018). As a reliable indicator of physiological response intensity (Boucsein, 2012), we recorded participants' skin conductance response (SCR) to the emotional images. We objectively quantified IAc by measuring the correlation between subjective ratings and SCRs (Baltazar et al., 2014; Hazem et al., 2017, 2018). Although there is no sensory system dedicated to perceiving SCRs per se, sympathetic activation leads to several correlated changes (heartbeat changes, pupil dilation, etc.), including SCRs that have been proven to reflect the level of arousal elicited by emotional stimuli and to correlate very well with subjective ratings of emotional reaction intensity (Boucsein, 2012; Lang \& Bradley, 2010). These characteristics make SCR a reliable and synthetic signal to confront to subjective ratings in our task, in order to obtain a measure of IAc, unspecific to a particular interoceptive modality. To identify brain regions associated with variation of IAc at the individual level, we fitted an fMRI model using the SCR values, the subjective ratings, and the product of the two (a proxy of participants' IAc, see (Anders et al., 2004)) as regressors on a trial-by-trial basis. Based on the results of previous studies investigating IAc as an individual trait via 
cardioception, we hypothesized that IAc would correlate with activity of the insula and medial frontal/dorsal cingulate gyrus.

\section{Materials and methods}

\section{Participants}

Thirty healthy adults (aged $20-29$ years, $M=22.7$ years, $S D=2.0 ; 15$ females) participated in the experiment. We based our sample size calculation on the results obtained in (Baltazar et al., 2014). The effect size f of social contact on IAc in this study was 0.42 . Based on this value, we computed a sample size of 30 for a power of 0.8 , at alpha .05 , using the G*Power 3 software (Faul et al., 2007).

All participants had normal or corrected-to-normal vision, were right-handed, French speakers and naive to the aim of the experiment. None of them had any neurological or psychiatric history. All participants provided written informed consent and were paid 80 euros for their participation. The whole procedure was approved by the local ethics committee and conformed to the World Medical Association's Declaration of Helsinki.

\section{Material}

The complete description of the material for this study can be found in Baltazar et al. (2014). Forty-eight emotionally arousing pictures from the International Affective Picture System (IAPS; (Lang et al., 2008)) were used to induce emotional experiences in the participants. We split them in four categories of 12 stimuli each (High Positive/Low Positive/High Negative/Low Negative), according to mean values of valence and arousal ratings obtained in a pre-test when designing the task for our former behavioral study (see Baltazar et al., 2014). Prior to arousal induction, participants were exposed to either a face displaying direct gaze, a face displaying averted gaze, or a fixation cross. Pictures from the Radboud Faces Database were used for the face stimuli (Langner et al., 2010). Since IAc has been shown to increase with social contact (see also Hazem et al., 2017 and Hazem et al., 
2018 for replications of the results), we used these context pictures in the present study to induce variations in IAc and favor the investigation of the neural bases of participants' IAc on a trial-by-trial basis (see Supplementary material).

\section{Experimental design and procedure}

An overview of the procedure can be found in Figure 1. The stimuli were projected on a screen with a resolution of $1024 \times 768$ pixels and placed $85 \mathrm{~cm}$ from the participants' eyes. The face stimuli covered a visual angle of $9^{\circ}$ horizontally and $15^{\circ}$ vertically. The emotional image covered a visual angle of $17^{\circ}$ horizontally and $12^{\circ}$ vertically. The fixation cross covered a visual angle of $2^{\circ} \times 2^{\circ}$.

Before the experiment, participants were told they would view emotional pictures, presented one by one. They were instructed to focus on the bodily changes that the emotional picture caused in them during its presentation and to evaluate their intensity from 0 (not at all intense) to 100 (very intense) on a visual analog scale. The way participants oriented their internal focus at each trial was left totally free. Participants were also informed that each emotional picture was to be preceded by either a cross or a face, and that they just had to fixate on the screen until the emotional picture appeared. Each trial was thus initiated by the context stimulus (i.e., a fixation cross, an averted face or a frontal face displaying eye contact), which appeared during $1500 \mathrm{~ms}$ in the center of the screen and was immediately followed by an emotional picture, which was displayed for $6 \mathrm{~s}$.

To disentangle the brain signals associated with emotional versus motor responses, we introduced a jitter in a pseudo logarithmic fashion between the offset of the emotional picture and the onset of the scale provided to record participants' evaluation (i.e. rating) at the end of each trial. Using 500ms steps, 50\% of trials included a jitter ranging from 200 to $2700 \mathrm{~ms}$, $31 \%$ of trials included a jitter ranging from 3000 to $4500 \mathrm{~ms}$, and $19 \%$ of trials included a jitter ranging from 4800 to $6300 \mathrm{~ms}$. This method ensured a high variability in jitter length while keeping the overall mean length of the experiment at a minimum (mean theoretical jitter 
duration $=2942 \mathrm{~ms}$ ). The scale remained onscreen until the participant gave his/her response, or after $6 \mathrm{~s}$ if no response was provided.

For $25 \%$ of the trials, a new screen appeared with the words "Side", "Front" or "Cross" displayed either on the left, center or right of the screen (the position of each word was randomly assigned by the computer program). Here, participants were instructed to recall which context picture had appeared before the emotional picture (i.e., either a fixation cross, an averted face or a frontal face displaying eye contact). This was an oddball task designed to ensure participants paid attention to the experimental images. Just like the rating scale, the presentation of this oddball response screen was self-paced and ended after $6 \mathrm{~s}$ if the participant did not provide a response.

Between trials, the screen remained black for 9 to $14 \mathrm{~s}$ (in increments of $0.5 \mathrm{~s}$ ) to ensure electrodermal activity returned to baseline levels. For each participant, the association between the context picture and the emotional stimuli was randomly determined with the constraint that every emotional picture $(\mathrm{N}=48)$ was seen once during the experiment and that each category of emotional picture (High Positive/Low Positive/High Negative/Low Negative) was seen in equal proportion in each context condition (Eye Contact/Averted Eyes/Fixation Cross). The 48 trials were divided into three sessions of 16 trials each, intermixed with a self-paced break.

(Figure 1 about here).

\section{Data acquisition and analyses}

\section{Ratings}

Using the Trackball Fiber Optic Response Pad (Current Designs Inc.), an fMRI-compatible device, participants used their right hand to scroll a ball that controls the cursor of the visual analog scale and select the correct answer for the oddball task. Once the cursor was appropriately positioned, participants clicked on the Trackball with their right thumb to 
validate their answer. The ratings were normalized per participant and per session to control for inter-participant and inter-session variability.

\section{Skin Conductance Responses (SCRs)}

Physiological responses were recorded using the MR-compatible acquisition system Biopac@ (GSR100C / LEAD108 / EL509). Electrodermal activity was recorded using two $\mathrm{Ag}-\mathrm{AgCl}$ electrodes filled with $0.5 \%$ saline unibase electrolyte that were attached to the palmar surface of the third phalanges of the index and middle fingers of the non-dominant hand. The raw SCR signal was recorded at a sampling rate of $5000 \mathrm{~Hz}$ and amplified and lowpass filtered online at a $1 \mathrm{~Hz}$ threshold. For subsequent analyses, the SCR was subsampled at $2 \mathrm{~Hz}$ using Matlab@. The SCR in response to emotional pictures was defined as the maximum change from baseline (computed during the second preceding the emotional picture onset) occurring between 1 and 6 s after the emotional picture onset. The first trial of each session was discarded from fMRI and skin conductance analyses to eliminate orienting responses that could occur at the beginning of a new session (see (Anders et al., 2004) for a similar procedure). Raw SCR values were transformed into $z$-scores per participant and per session to normalize the data.

\section{fMRI}

Functional magnetic resonance imaging (fMRI) was performed on a Siemens 3T wholebody scanner (Siemens MAGNETOM Verio) equipped with a 32-channel head coil and a custom-built head holder for movement reduction. We used a T2-weighted gradient echo planar imaging (EPI) sequence with a repetition time (TR) of 2220ms, an echo time (TE) of $30 \mathrm{~ms}$, and a field of view (FOV) of $198 \mathrm{~mm}$. Each volume consisted of 403 -mm axial slices and was acquired in a sequential ascending order. Because the presentation of both the scale and the secondary task were self-paced, experiment duration varied slightly between participants (mean number of scans $=589 \pm 11$; mean duration $=22 \mathrm{mn} \pm 24 \mathrm{~s}$ ). Each fMRI 
functional acquisition sequence began with a short scanner equilibration phase equivalent to 3 TRs. The corresponding volumes were not stored and therefore not included in the analyses.

Data were preprocessed and analyzed using the Statistical Parametric Mapping software (SPM12; Welcome Trust Center for Neuroimaging, London, UK, 2014). Preprocessing included the following steps: (1) slice timing, (2) realignment to the first scan by rigid body transformations to correct for head movements, (3) co-registration of structural images to corresponding mean EPI images, (4) segmentation, (5) normalization of individual EPI images to MNI space using the forward deformation parameters and (6) spatial smoothing using an $8 \mathrm{~mm}$ full width at half maximum Gaussian kernel. A high-pass filter with a cut-off period of 128s was applied to remove low-frequency-drifts. Voxels were resampled from $3 \times 3 \times 3 \mathrm{~mm}$ to $2 \times 2 \times 2 \mathrm{~mm}$ during normalization.

We first fitted a standard general linear model (GLM) to each participant. To analyze the neural bases of participants' IAc on a trial-by-trial basis with sufficient statistical power, we collapsed events regardless of the valence of the emotional images and of the context pictures. The first regressor (Simple) modeled a $7.5 \mathrm{sec}$ interval as a boxcar function time-locked to the onset of the context and ending with the offset of the emotional picture ${ }^{1}$. Regressors 2 to 4 modeled parametric modulations of the first regressor by the normalized SCR values (SCR), the normalized ratings of emotional intensity (Ratings) and the pairwise product of the two (SCR*Ratings), respectively. The pairwise product of SCR*Ratings was used as a proxy of trial-by-trial correlations between SCRs and Ratings (see (Anders et al., 2004) for a similar approach). When averaged across each subject, it correlates almost perfectly with the mean correlations computed between SCRs and ratings $(\mathrm{R}=0.997)$, which confirms the view that

\footnotetext{
${ }^{1}$ In the absence of a jitter between the contextual and the emotional pictures, the two events were correlated and therefore modeled together as a single event.
} 
such a pairwise product is a good index of trial-by-trial IAc. The fifth regressor corresponded to the behavioral responses provided by the participants. It modeled a variable time interval as a boxcar function time-locked to the onset of the visual analog scale and ending with participants' ratings or responses on the oddball task, which occurred on $25 \%$ of trials. The sixth regressor included the trials for which no rating was provided (mean per participant $=$ $1.1 \pm 1.1$ ) and the first trial of each session (see SCRs section). Finally, the GLM matrix also included the six motion correction parameters as regressors of no interest.

In the second level group analysis, we computed one sample t-test statistical maps for each of the three regressors of interest: the parametric modulation of normalized SCR values (Regressor 2), the parametric modulation of normalized ratings of emotional intensity (Regressor 3), and the parametric modulation of the pairwise product of the two (SCR*Ratings; Regressor 4). Positive and negative activations were investigated for each model. To protect against false-positive activations, significance thresholds of $P<.001$ at the voxel level (uncorrected) and $P<.05$ at the cluster level (Family Wise Error - FWEcorrected) were applied. We used bspmview (Spunt, 2016) and the AAL3 Toolbox (Rolls et al., 2015, 2020; Tzourio-Mazoyer et al., 2002) to visualize and identify anatomical regions.

\section{Results}

The mean ratings were approximately situated at the middle of the visual analog scale $(M=$ 47.2, SD = 10.4), and the mean SCRs were well above the minimum threshold usually used in SCR studies $(M=0.23 \mu \mathrm{S}, S D=0.27$; minimum threshold $=0.01$ to $0.05 \mu \mathrm{S}$; (Boucsein, 2012)). The mean correlation between Ratings and SCRs was significantly different from 0 $\left(M=0.24 \mu \mathrm{S}, S D=0.21 ; t_{29}=6.25, P<.001\right)$, just as the product between SCRs and Ratings $\left(M=0.21 \mu \mathrm{S}, S D=0.18 ; t_{29}=6.60, P<.001\right)$. These results show that the emotional pictures used in this fMRI paradigm did elicit physiological changes in our participants, and that these changes were perceived by the participants. 
As a sanity check, we report here the results for positive BOLD activations in response to picture presentation. They are displayed in Table 1. As expected, they reveal strong activations in visual areas (Fusiform, Occipital and Temporal gyri) and emotional appraisal areas (Insula, Amygdala, Thalamus and Cingulate Cortex).

(Table 1 about here).

Whole-brain fMRI analyses revealed several brain areas where activity positively covaried with SCR levels. This network involved the anterior dorsal and pregenual parts of the anterior cingulate cortex (ACC), the left amygdala and hippocampus region and temporo-occipital brain regions. The brain regions where activity increased with subjective ratings were the ventromedial pre-frontal cortex (vmPFC), the superior colliculus, the thalamus, the right amygdala and hippocampus, as well as the fusiform gyrus and the middle temporal and occipital gyri. Finally, we observed a negative correlation between SCR*Ratings (i.e., IAc) and activity in the dorsomedial pre-frontal cortex (dmPFC). No voxel survived the statistical threshold when considering activities that decreased with SCR or with Ratings, nor when considering activities that increased with SCR*Ratings. These fMRI results are presented in Table 2 and Figure 2.

(Table 2 about here)

(Figure 2 about here)

\section{Discussion}

So far, studies investigating brain mechanisms of IAc mainly focused on IAc as an individual trait, and on cardioception as the interoceptive signal, which limits the generalizability of the findings. In the present study, we explored the neural correlates of broader (i.e., modality-unspecific) and more ecological IAc in healthy adult participants. Using a parametric approach to map the correlation between IAc and brain activity on a trialby-trial basis, we revealed brain areas subtending intra-individual variations of IAc, as they 
can occur in daily life. We found that activity in dorsomedial pre-frontal cortex (dmPFC) is negatively correlated with IAc. The more activity in the dmPFC, the more physiological responses elicited by emotional pictures were mismatched with subjective ratings about the intensity of these responses (corresponding to a low IAc).

To our knowledge, our study is the first to assess the neural basis of IAc on a trial-by-trial basis and without asking participants to focus on a particular interoceptive signal. These two aspects most likely account for the different results reported in previous studies, which computed correlations at the group level and focused on cardioception (Critchley et al., 2004; Pollatos et al., 2007). These previous studies reported positive correlations between cardioceptive accuracy and insular activity (Critchley et al., 2004; Pollatos et al., 2007). The discrepancy with our own results may suggest that the insula is involved in IAc only when related to cardioception or, at least, to a specific interoceptive signal. Since participants were free to rely on a wide range of interoceptive sensations in our study, activations of the insula could vary substantially depending on the interoceptive signals used by participants at each trial, which may have prevented robust activity of the insula to be detected in our results. Furthermore, previous studies applied a masking procedure that limited the analyses to brain areas associated with cardioceptive attentiveness (i.e., the capacity to focus attention on cardioception). According to the meta-analysis by Schulz (2016), the main brain correlate of cardioceptive attentiveness is indeed the insula, which aims at triggering and/or maintaining attentiveness to a particular interoceptive signal (see e.g., (Wang et al., 2019) for involvement of the insula in attentiveness to the breath). In sum, our results show that the insula is not the most relevant candidate subtending intra-individual modulations of IAc, when approached as a modality unspecific ability.

Instead of a positive correlation of IAc with the anterior insula, we found a negative correlation of IAc with the dmPFC. The pattern of the results suggests that the dmPFC processes the mismatch between objective (i.e. SCR) and subjective arousal (i.e. ratings of 
arousal), which is reminiscent of predictive coding accounts of interoceptive accuracy (Petzschner et al., 2021). The predictive coding framework proposes that the brain is constantly generating internal models of the world, where top-down predictions of sensory inputs (priors) are compared to actual sensory inputs. These comparisons then yield prediction error signals that are used to update predictions and/or select the appropriate action in order to reduce the discrepancy between predictions and sensory signals (i.e. active inference; (Friston, 2009)). This framework has been applied to interoception, leading to the rise of several computational models (Ainley et al., 2016; Barrett \& Simmons, 2015; Petzschner et al., 2021). Our data bring new empirical evidence supporting this framework and are consistent with a large literature demonstrating the involvement of the dmPFC in error processing in a variety of tasks and contexts (Alexander \& Brown, 2014; Jiang et al., 2018; Nee et al., 2011; Zarr \& Brown, 2016). To our knowledge, the dmPFC does not directly receive interoceptive inputs, suggesting that it is situated at higher hierarchical levels and is more specifically involved in minimizing error in the context of a mismatch between ascending sensory inputs and descending predictions (see (Jiang et al., 2018) for a similar interpretation in the context of cognitive control).

Error processing probably occurred outside of conscious experience in the present study, as no feedback was provided to the participants regarding their interoceptive accuracy. Accordingly, the dmPFC is also involved in placebo analgesia, another case of mismatch between physiological signals and their cognitive appraisal that occurs without awareness, with deactivation of the dmPFC when placebo effects occur (Ashar et al., 2017; Wager \& Atlas, 2015). However, it is possible that our task, which requires participants to focus explicitly on bodily changes, boosted or altered processes related to IAc that seemingly occur subconsciously in daily life (e.g., deciding when to stop food and water intake on a lunch break). However, to date, there is no reported method in the field to capture IAc exempt from such explicit processes as all studies ask participants to focus on one bodily signal or another. 
Moreover, the strength of our method was to use a parametric approach for identifying brain correlates of IAc (computed here as the product of SCRs*Ratings) at the individual level. With this method we were able to introduce SCRs and Ratings obtained trial by trial in the statistical model, allowing us to distinguish between brain activity related solely to SCR/body related processing from brain activity specifically related to IAc. This most likely limited the impact of explicit processes on our main result.

Regarding patterns of brain activity that correlated with ratings or SCRs, Lang et al. (2010) noted that greater activation of visual centers is consistently observed when people view emotional compared with neutral pictures. They also reported that average ratings of emotional arousal of the IAPS (the database that we used in the present experiment) correlated with activity in a large part of the inferotemporal cortex (encompassing fusiform gyrus and other visual areas). Accordingly, we found that the activity of several visual centers correlates with ratings (fusiform gyrus, middle temporal and occipital gyri) and/or with SCRs (superior temporal sulcus, middle occipital gyrus). These activities must be related to the visual properties of our task and fit embodiment theories of emotion, according to which emotional processing is dependent on perceptual resources (Winkielman et al., 2015). The fact that ratings correlated with activity of the amygdala and the vmPFC is in line with the view that these brain structures are interconnected and underpin value-based decision making (e.g. (Dixon et al., 2017)), thus corroborating the validity of our regression approach. In the same vein, we found that SCRs correlated with activity in the amygdala, the hippocampus and the ACC, which have all been consistently implicated in the cerebral network of emotional processing and/or physiological arousal (e.g. (Brooks et al., 2012; Zhang et al., 2014)).

The full cascade of mechanisms subtending the updating of interoceptive predictions and the ensuing improvement of IAc remains to be resolved. This issue is particularly important to address, as current models of interoception converge toward the view that deficits in interoception lead to the emergence of cognitive and psychological disorders (Duquette, 2017; 
Murphy et al., 2017; Quadt et al., 2018). However, understanding these mechanisms represents a challenge due to the fact that the brain integrates information from multiple systems simultaneously to represent particular states (Khalsa \& Lapidus, 2016; Petzschner et al., 2021). So far, studies investigating brain mechanisms of IAc exclusively focused on cardioception (and sometimes on breathing), which limits the generalizability of these findings. An expanded approach that assesses multiple interoceptive organ systems would be more appropriate but is very difficult to apply (Khalsa \& Lapidus, 2016; Petzschner et al., 2021). Here, we asked participants to assess overall interoceptive reactions. They were free to rely on any bodily reactions to make their evaluation and we used SCRs as a synthetic index of those reactions. We believe that such an approach represents a helpful compromise. Other measures of physiological arousal could also be interesting in this regard, such as pupil dilation (Kret \& Sjak-Shie, 2019), which is less prone to habituation than SCRs (Isen et al., 2013) and thus allows for more extended testing.

Our results highlight that our paradigm may have practical utility in the clinical domain. Indeed, our data support the predictive coding framework suggesting that a deficit in IAc reveals an impairment in processing a mismatch between actual interoceptive signals and predictions. Our paradigm may help diagnose conditions involving problems with error detection and regulation, including autism, anxiety or alexithymia, and opens new avenues for clinical intervention via interoceptive training and feedback (Seth \& Friston, 2016). Beyond providing a new way to detect interoception deficits, our paradigm may be used to provide biofeedback of brain error signals to help the system reduce error, for instance by updating predictions or by active inference (Petzschner et al., 2021). However, future investigations should first elucidate whether disturbed patterns of physiological reactivity reported in conditions like autism, anxiety or alexithymia (e.g. (Hyde et al., 2019; Peasley-Miklus et al., 2016; Zantinge et al., 2019)) are the primary cause of deficits in error processing, or if such conditions are associated with impairments of IAc per se. 


\section{Conclusion}

For the first time, we used an ecological task under fMRI to investigate the neural bases of intra-individual variation of IAc, unspecific to a particular interoceptive modality. Previous results using heartbeat-focused tasks to investigate brain correlates of IAc highlighted the central role of the insula. We show that IAc also relies on general mechanisms of error processing, unspecific to interoception, which are mediated by the activity of the dorsomedial prefrontal cortex.

\section{Acknowledgements}

This work was supported by the European Research Council (advanced grant, $\mathrm{n}^{\circ} 269616$ ) and by the French National Research Agency (Grant numbers ANR-13-JSH2-0001-01). We thank the CENIR team for their support in collecting and analyzing the data.

\section{Disclosure Statement}

The authors have no conflict of interest to declare.

\section{Author Contributions}

LC, JG, JLP and MB designed the study. MB collected and analyzed the data. LC, MB, JG, JLP and MMG wrote the manuscript.

\section{Data Availability Statements}

Publicly available datasets were analyzed in this study. This data can be found here: https://osf.io/8w63q/.

\section{Abbreviations}

$\mathrm{ACC}=$ Anterior Cingulate Cortex

ACCpre $=$ Anterior Cingulate Cortex pregenual

ACCsub $=$ Anterior Cingulate Cortex subgenual

ACCsup $=$ Anterior Cingulate Cortex supracallosal

$\operatorname{adACC}=$ anterior dorsal Anterior Cingulate Cortex 
$\mathrm{AMYG}=$ Amygdala

BOLD = Blood Oxygen Level Dependent

$\mathrm{dmPFC}=$ dorsomedial Pre-Frontal Cortex

EPI = Echo Planar Imaging

$\mathrm{FG}=$ Fusiform Gyrus

fMRI = functional Magnetic Resonance Imaging

$\mathrm{FOV}=$ Field Of View

FWE $=$ Family Wise Error

GLM = General Linear Model

HIP = Hippocampus

IAc $=$ Interoceptive Accuracy

IAPS = International Affective Picture System

MNI $=$ Montreal Neurological Institute

MTG = Medial Temporal Gyrus

ROI $=$ Region Of Interest

SCR $=$ Skin Conductance Response

$\mathrm{SN}=$ Substantia Nigra

SPM $=$ Statistical Parametric Mapping

$\mathrm{TE}=$ Echo Time

THA $=$ Thalamus

$\mathrm{TR}=$ Repetition Time

vmPFC $=$ ventromedial Pre-Frontal Cortex

\section{References}

Ainley, V., Apps, M. A. J., Fotopoulou, A., \& Tsakiris, M. (2016). 'Bodily precision' : A predictive coding account of individual differences in interoceptive accuracy. 
Philosophical Transactions of the Royal Society of London. Series B, Biological Sciences, 371(1708). https://doi.org/10.1098/rstb.2016.0003

Ainley, V., Maister, L., Brokfeld, J., Farmer, H., \& Tsakiris, M. (2013). More of myself : Manipulating interoceptive awareness by heightened attention to bodily and narrative aspects of the self. Consciousness and Cognition, 22(4), 1231- 1238. https://doi.org/10.1016/j.concog.2013.08.004

Alexander, W. H., \& Brown, J. W. (2014). A general role for medial prefrontal cortex in event prediction. Frontiers in Computational Neuroscience, 8. https://doi.org/10.3389/fncom.2014.00069

Anders, S., Lotze, M., Erb, M., Grodd, W., \& Birbaumer, N. (2004). Brain activity underlying emotional valence and arousal: A response-related fMRI study. Human Brain Mapping, 23(4), 200- 209. https://doi.org/10.1002/hbm.20048

Ashar, Y. K., Chang, L. J., \& Wager, T. D. (2017). Brain Mechanisms of the Placebo Effect : An Affective Appraisal Account. Annual Review of Clinical Psychology, 13, 73- 98. https://doi.org/10.1146/annurev-clinpsy-021815-093015

Baltazar, M., Hazem, N., Vilarem, E., Beaucousin, V., Picq, J.-L., \& Conty, L. (2014). Eye contact elicits bodily self-awareness in human adults. Cognition, 133(1), 120- 127. https://doi.org/10.1016/j.cognition.2014.06.009

Barrett, L. F., \& Simmons, W. K. (2015). Interoceptive predictions in the brain. Nature Reviews Neuroscience, 16(7), 419- 429. https://doi.org/10.1038/nrn3950

Boucsein, W. (2012). Electrodermal activity, 2nd ed. Springer Science + Business Media. https://doi.org/10.1007/978-1-4614-1126-0

Brooks, S. J., Savov, V., Allzén, E., Benedict, C., Fredriksson, R., \& Schiöth, H. B. (2012). Exposure to subliminal arousing stimuli induces robust activation in the amygdala, hippocampus, anterior cingulate, insular cortex and primary visual cortex : A 
systematic meta-analysis of fMRI studies. NeuroImage, 59(3), 2962- 2973.

https://doi.org/10.1016/j.neuroimage.2011.09.077

Carvalho, G. B., \& Damasio, A. (2021). Interoception and the origin of feelings : A new synthesis. BioEssays: News and Reviews in Molecular, Cellular and Developmental Biology, e2000261. https://doi.org/10.1002/bies.202000261

Caseras, X., Murphy, K., Mataix- Cols, D., López- Solà, M., Soriano- Mas, C., Ortriz, H., Pujol, J., \& Torrubia, R. (2013). Anatomical and functional overlap within the insula and anterior cingulate cortex during interoception and phobic symptom provocation. Human Brain Mapping, 34(5), 1220- 1229. https://doi.org/10.1002/hbm.21503

Chen, W. G., Schloesser, D., Arensdorf, A. M., Simmons, J. M., Cui, C., Valentino, R., Gnadt, J. W., Nielsen, L., Hillaire-Clarke, C. St., Spruance, V., Horowitz, T. S., Vallejo, Y. F., \& Langevin, H. M. (2021). The Emerging Science of Interoception : Sensing, Integrating, Interpreting, and Regulating Signals within the Self. Trends in Neurosciences, 44(1), 3- 16. https://doi.org/10.1016/j.tins.2020.10.007

Craig, A. D. B. (2009). How do you feel--now? The anterior insula and human awareness. Nature Reviews. Neuroscience, 10(1), 59- 70. https://doi.org/10.1038/nrn2555

Critchley, H. D., Wiens, S., Rotshtein, P., Ohman, A., Dolan, R. J., Öhman, A., \& Dolan, R. J. (2004). Neural systems supporting interoceptive awareness. Nature Neuroscience, 7(2), 189- 195. https://doi.org/10.1038/nn1176

Dan-Glauser, E. S., \& Gross, J. J. (2013). Emotion regulation and emotion coherence : Evidence for strategy-specific effects. Emotion, 13(5), 832.

Desmedt, O., Luminet, O., \& Corneille, O. (2018). The heartbeat counting task largely involves non-interoceptive processes : Evidence from both the original and an adapted counting task. Biological Psychology, 138, 185- 188.

https://doi.org/10.1016/j.biopsycho.2018.09.004 
Dixon, M. L., Thiruchselvam, R., Todd, R., \& Christoff, K. (2017). Emotion and the prefrontal cortex : An integrative review. Psychological bulletin, 143(10), 1033.

Duquette, P. (2017). Increasing Our Insular World View : Interoception and Psychopathology for Psychotherapists. Frontiers in Neuroscience, 11, 135. https://doi.org/10.3389/fnins.2017.00135

Durlik, C., Brown, G., \& Tsakiris, M. (2014). Enhanced interoceptive awareness during anticipation of public speaking is associated with fear of negative evaluation. Cognition \& Emotion, 28(3), 530- 540. https://doi.org/10.1080/02699931.2013.832654

Faul, F., Erdfelder, E., Lang, A.-G., \& Buchner, A. (2007). G*Power 3 : A flexible statistical power analysis program for the social, behavioral, and biomedical sciences. Behavior Research Methods, 39(2), 175- 191. https://doi.org/10.3758/BF03193146

Ferentzi, E., Drew, R., Tihanyi, B. T., \& Köteles, F. (2018). Interoceptive accuracy and body awareness-Temporal and longitudinal associations in a non-clinical sample. Physiology \& Behavior, 184, 100- 107. https://doi.org/10.1016/j.physbeh.2017.11.015

Friston, K. (2009). The free-energy principle : A rough guide to the brain? Trends in Cognitive Sciences, 13(7), 293- 301. https://doi.org/10.1016/j.tics.2009.04.005

Garfinkel, S. N., Seth, A. K., Barrett, A. B., Suzuki, K., \& Critchley, H. D. (2015). Knowing your own heart : Distinguishing interoceptive accuracy from interoceptive awareness. Biological Psychology, 104, 65- 74. https://doi.org/10.1016/j.biopsycho.2014.11.004

Garfinkel, S. N., Tiley, C., O’Keeffe, S., Harrison, N. A., Seth, A. K., \& Critchley, H. D. (2016). Discrepancies between dimensions of interoception in autism : Implications for emotion and anxiety. Biological Psychology, 114, 117- 126.

https://doi.org/10.1016/j.biopsycho.2015.12.003 
Hazem, N., Beaurenaut, M., George, N., \& Conty, L. (2018). Social Contact Enhances Bodily Self-Awareness. Scientific Reports, 8(1), 4195. https://doi.org/10.1038/s41598-01822497-1

Hazem, N., George, N., Baltazar, M., \& Conty, L. (2017). I know you can see me : Social attention influences bodily self-awareness. Biological Psychology, 124, 21- 29. https://doi.org/10.1016/j.biopsycho.2017.01.007

Howell, D. C. (2001). Statistical Methods for Psychology (5th éd.). Wadsworth Publishing. Hyde, J., Ryan, K. M., \& Waters, A. M. (2019). Psychophysiological Markers of Fear and Anxiety. Current Psychiatry Reports, 21(7), 56. https://doi.org/10.1007/s11920-019$1036-\mathrm{x}$

Isen, J. D., Iacono, W. G., \& Malone, S. M. (2013). Characterizing electrodermal response habituation : A latent class approach with application to psychopathology. Psychophysiology, 50(10), 954- 962. https://doi.org/10.1111/psyp.12080

Jiang, J., Wagner, A. D., \& Egner, T. (2018). Integrated externally and internally generated task predictions jointly guide cognitive control in prefrontal cortex. ELife, 7. https://doi.org/10.7554/eLife.39497

Khalsa, S. S., \& Lapidus, R. C. (2016). Can Interoception Improve the Pragmatic Search for Biomarkers in Psychiatry? Frontiers in Psychiatry, 7. https://doi.org/10.3389/fpsyt.2016.00121

Kret, M. E., \& Sjak-Shie, E. E. (2019). Preprocessing pupil size data : Guidelines and code. Behavior Research Methods, 51(3), 1336- 1342. https://doi.org/10.3758/s13428-018$1075-y$

Lang, P. J., \& Bradley, M. M. (2010). Emotion and the motivational brain. Biological Psychology, 84(3), 437- 450. https://doi.org/10.1016/j.biopsycho.2009.10.007 
Lang, P. J., Bradley, M. M., \& Cuthbert, B. N. (2008). International affective picture system (IAPS) : Affective ratings of pictures and instruction manual. (Technical Report A-8). University of Florida.

Langner, O., Dotsch, R., Bijlstra, G., Wigboldus, D. H., Hawk, S. T., \& Van Knippenberg, A. D. (2010). Presentation and validation of the Radboud Faces Database. Cognition and emotion, 24(8), 1377- 1388.

Murphy, J., Brewer, R., Catmur, C., \& Bird, G. (2017). Interoception and psychopathology : A developmental neuroscience perspective. Developmental Cognitive Neuroscience, 23, 45- 56. https://doi.org/10.1016/j.den.2016.12.006

Murphy, J., Millgate, E., Geary, H., Ichijo, E., Coll, M.-P., Brewer, R., Catmur, C., \& Bird, G. (2018). Knowledge of resting heart rate mediates the relationship between intelligence and the heartbeat counting task. Biological Psychology, 133, 1- 3. https://doi.org/10.1016/j.biopsycho.2018.01.012

Nee, D. E., Kastner, S., \& Brown, J. W. (2011). Functional heterogeneity of conflict, error, task-switching, and unexpectedness effects within medial prefrontal cortex. NeuroImage, 54(1), 528- 540. https://doi.org/10.1016/j.neuroimage.2010.08.027

Peasley-Miklus, C. E., Panayiotou, G., \& Vrana, S. R. (2016). Alexithymia predicts arousalbased processing deficits and discordance between emotion response systems during emotional imagery. Emotion (Washington, D.C.), 16(2), 164- 174. https://doi.org/10.1037/emo0000086

Petzschner, F. H., Garfinkel, S. N., Paulus, M. P., Koch, C., \& Khalsa, S. S. (2021). Computational Models of Interoception and Body Regulation. Trends in Neurosciences, 44(1), 63- 76. https://doi.org/10.1016/j.tins.2020.09.012

Pollatos, O., Schandry, R., Auer, D. P., \& Kaufmann, C. (2007). Brain structures mediating cardiovascular arousal and interoceptive awareness. Brain Research, 1141, 178- 187. https://doi.org/10.1016/j.brainres.2007.01.026 
Quadt, L., Critchley, H. D., \& Garfinkel, S. N. (2018). The neurobiology of interoception in health and disease. Annals of the New York Academy of Sciences, 1428(1), 112- 128. https://doi.org/10.1111/nyas.13915

Ring, C., Brener, J., Knapp, K., \& Mailloux, J. (2015). Effects of heartbeat feedback on beliefs about heart rate and heartbeat counting : A cautionary tale about interoceptive awareness. Biological Psychology, 104, 193- 198. https://doi.org/10.1016/j.biopsycho.2014.12.010

Rolls, E. T., Huang, C.-C., Lin, C.-P., Feng, J., \& Joliot, M. (2020). Automated anatomical labelling atlas 3. NeuroImage, 206, 116189. https://doi.org/10.1016/j.neuroimage.2019.116189

Rolls, E. T., Joliot, M., \& Tzourio-Mazoyer, N. (2015). Implementation of a new parcellation of the orbitofrontal cortex in the automated anatomical labeling atlas. NeuroImage, 122, 1- 5. https://doi.org/10.1016/j.neuroimage.2015.07.075

Schulz, S. M. (2016). Neural correlates of heart-focused interoception : A functional magnetic resonance imaging meta-analysis. Philosophical Transactions of the Royal Society of London. Series B, Biological Sciences, 371(1708). https://doi.org/10.1098/rstb.2016.0018

Seth, A. K., \& Friston, K. J. (2016). Active interoceptive inference and the emotional brain. Philosophical Transactions of the Royal Society B: Biological Sciences, 371(1708), 20160007. https://doi.org/10.1098/rstb.2016.0007

Spunt, B. (2016). Bspmview. https://spunt.github.io/software/bspmview/

Tzourio-Mazoyer, N., Landeau, B., Papathanassiou, D., Crivello, F., Etard, O., Delcroix, N., Mazoyer, B., \& Joliot, M. (2002). Automated anatomical labeling of activations in SPM using a macroscopic anatomical parcellation of the MNI MRI single-subject brain. NeuroImage, 15(1), 273- 289. https://doi.org/10.1006/nimg.2001.0978 
Wager, T. D., \& Atlas, L. Y. (2015). The neuroscience of placebo effects : Connecting context, learning and health. Nature Reviews. Neuroscience, 16(7), 403- 418. https://doi.org/10.1038/nrn3976

Wang, X., Wu, Q., Egan, L., Gu, X., Liu, P., Gu, H., Yang, Y., Luo, J., Wu, Y., Gao, Z., \& Fan, J. (2019). Anterior insular cortex plays a critical role in interoceptive attention. eLife, 8, e42265. https://doi.org/10.7554/eLife.42265

Ward, D., \& Stapleton, M. (2012). Es Are Good : Cognition as Enacted, Embodied, Embedded, Affective and Extended. In Consciousness in Interaction : The Role of the Natural and Social Context in Shaping Consciousness (F. Paglieri, p. 89- 104). John Benjamins Publishing.

Winkielman, P., Niedenthal, P., Wielgosz, J., Eelen, J., \& Kavanagh, L. C. (2015). Embodiment of cognition and emotion. In APA handbook of personality and social psychology, Volume 1 : Attitudes and social cognition (p. 151- 175). American Psychological Association. https://doi.org/10.1037/14341-004

Wittkamp, M. F., Bertsch, K., Vögele, C., \& Schulz, A. (2018). A latent state-trait analysis of interoceptive accuracy. Psychophysiology, 55(6), e13055. https://doi.org/10.1111/psyp.13055

Zamariola, G., Maurage, P., Luminet, O., \& Corneille, O. (2018). Interoceptive accuracy scores from the heartbeat counting task are problematic : Evidence from simple bivariate correlations. Biological Psychology, 137, 12- 17. https://doi.org/10.1016/j.biopsycho.2018.06.006

Zantinge, G., van Rijn, S., Stockmann, L., \& Swaab, H. (2019). Concordance between physiological arousal and emotion expression during fear in young children with autism spectrum disorders. Autism: The International Journal of Research and Practice, 23(3), 629- 638. https://doi.org/10.1177/1362361318766439 
Zarr, N., \& Brown, J. W. (2016). Hierarchical error representation in medial prefrontal cortex.

NeuroImage, 124, 238- 247. https://doi.org/10.1016/j.neuroimage.2015.08.063

Zhang, S., Hu, S., Chao, H. H., Ide, J. S., Luo, X., Farr, O. M., \& Li, C. R. (2014).

Ventromedial prefrontal cortex and the regulation of physiological arousal. Social

Cognitive and Affective Neuroscience, 9(7), 900- 908.

https://doi.org/10.1093/scan/nst064 


\section{SUPPLEMENTARY: Data analyses and results}

Context pictures, depicting either a face with direct gaze, a face with averted gaze, and a fixation cross were used to induce variations in IAc (see Baltazar et al., 2014; Hazem et al., 2017; Hazem et al., 2018) and favor the investigation of the neural bases of participants' IAc on a trial-by-trial basis. This variable was not introduced in the fMRI model for 2 reasons. Firstly, this would have led to a small number of trials per experimental condition (16 trials per Context) and poor statistical power. On the other hand, increasing the number of trials was risky, as SCR is well known to induce habituation (e.g. (Isen et al., 2013)). Secondly, the onset of both the context and the emotional picture were not decorrelated (i.e., no jitter was introduced between the two events). This was because the duration of the effect of eye contact remains unknown. One may expect it to vanish quite rapidly after the eye contact has occurred. Here we report behavioral analyses and results related to this variable.

First, the oddball task controlling that participants actually paid attention to the context picture showed high mean accuracy $(M=86.9 \%, S D=8.7 \%)$.

To analyze whether the physiological responses evoked by the emotional pictures were correlated to participants' ratings, we calculated intra-subject correlations for each participant across all images for each context, and performed Fisher's $r$-to- $z$ transformations on the $R$ values to normalize them (Howell, 2001). The Fisher's zs were then submitted to a repeated measures ANOVA with Context (Cross/Averted Eyes/Eye Contact) as the main withinsubject factor (see (Baltazar et al., 2014; Dan-Glauser \& Gross, 2013) for a similar method).

We conducted ANOVAs with Context (Cross/Averted Eyes/Eye Contact) as a within-subject factor on Rating, SCR and mean correlations between SCRs and ratings (Fisher's zs). As previously reported, context did not modulate $\operatorname{SCR}$ activity $\left(F_{2,58}=0.51, P=.61\right)$. In particular, Eye Contact did not elicit greater physiological arousal. Similarly, context also did 
not affect the ratings $\left(F_{2,58}=0.99, P=.38\right)$. Context did not significantly affect the correlations between SCRs and ratings in this sample $\left(F_{2,58}=0.88, P=.42\right)$ (see Table $\left.\mathrm{S} 1\right)$.

Of interest, the mean correlation between SCRs and Ratings $(M=0.245, S D=0.214)$ was significantly higher than in our previous study $\left(M=0.075, S D=0.250 ; F_{1,56}=12.16, P<\right.$ $.001 ; \eta^{2}=.18$; Baltazar et al., 2014), suggesting that the fMRI environment may have favored interoceptive accuracy. This may explain why, and contrary to our previous experiments (Baltazar et al., 2014; Hazem et al., 2017), no effect of Context was observed on the mean correlations between SCRs and ratings.

\section{$\underline{\text { Table S1 }}$}

\begin{tabular}{lccc}
\hline & Cross & Averted Eyes & Eye Contact \\
\hline Intensity ratings & $47.6 \pm 10.9$ & $47.9 \pm 11.8$ & $46.4 \pm 10.2$ \\
Skin Conductance Responses (SCR) & $0.26 \pm 0.34$ & $0.21 \pm 0.28$ & $0.24 \pm 0.33$ \\
Correlations between ratings and SCR & $0.20 \pm 0.29$ & $0.26 \pm 0.28$ & $0.29 \pm 0.33$
\end{tabular}

Effects of context on ratings, physiological activity, and correlations between mean physiological responses and ratings. All values are expressed as means \pm standard errors. Skin conductance responses (SCRs) are expressed in $\mu \mathrm{S}$. Correlations are expressed in Fisher's $z$.

For exploratory purposes, we built an ROI aggregating Left and Right Insula areas from the AAL3 toolbox (Total number of voxels = 3628; (Rolls et al., 2020)). When applying this ROI, no significant positive or negative activations were found for the SCR * Rating parametric modulator (even with a $\mathrm{p}<.05$ FWE corrected at the cluster level and an uncorrected $\mathrm{p}<.001$ at the peak level). 